\title{
MODELO PARA EL DESARROLLO DE GESTIÓN DEL CONOCIMIENTO EN EL ÁREA DE INVESTIGACIÓN
}

MODEL FOR THE KNOWLEDGE MANAGEMENT DEVELOPMENT IN THE RESEARCH AREA

\section{Luisa Elena Salcedo Guzmán* Zelma Wong Torres**}

Docentes Principales de la Facultad de Ciencias Contables

Universidad Nacional Mayor de San Marcos-UNMSM

Lima-Perú

[Recepción:Setiembre de 2012/ Conformidad: Octubre de 2012]

\section{RESUMEN}

Todas las organizaciones, independientemente de que pertenezcan al sector público o privado, deben tener las herramientas necesarias para enfrentar los cambios del entorno, la Gerencia del Conocimiento, que se basa en la medición y evaluación del Capital Intelectual, ofrece la metodología adecuada para este objetivo y es una herramienta administrativa adaptable a cualquier tipo de organización.Sin embargo, resulta difícil aplicar esta herramienta que ha sido diseñada para empresas privadas, en organizaciones públicas, más aún en las universidades nacionales.

El presente trabajo busca presentar la aplicación de un modelo de gestión teórico para contribuir al desarrollo de la producción científica dentro de la Universidad Nacional Mayor de San Marcos, dicho modelo está basado en la Gestión de Conocimiento y adaptado a las características propias de la institución de educación superior pública. Este artículo es el resultado de revisiones bibliográficas y forma parte del proyecto de investigación "Modelo Gerencial para el desarrollo de proyectos aplicativos".

Palabras clave: Conocimiento, Capital Intelectual, Gestión del conocimiento, Propiedad Intelectual.

\begin{abstract}
All organizations, whether they belong to the public or private sector, should have the necessary tools to meet the changing environment. The Knowledge Management, which is based on the measurement and evaluation of Intellectual Capital, provides the methodology for this purpose and is a management tool adaptable to any type of organization. However, it is difficult to apply this tool that has been designed for private companies, to the public institutions, especially in national universities.
\end{abstract}

This paper seeks to present the way to apply of a theoretical model management to contribute to the development of scientific production in the Universidad Nacional Mayor de San Marcos, the model is based on Knowledge Management and was adapted to the characteristics of the institution public higher education.

This article is the result of literature reviews and is part of the research project "Management model for development of application projects"

Keywords: Knowledge, Intellectual Capital, Knowledge management, Intellectual property.

* Egresada de Postgrado, Doctorante- UNMSM. CPCC. Docente Investigadora de la UNMSM. Email: luisalcedog@yahoo.es

** Doctora en Contabilidad y Magister en Control Interno. C.P.C.C. Docente Investigadora de la UNMSM. Email: zwont@hotmail.com 


\section{INTRODUCCION}

Para gestionar la tecnología, el conocimiento, el capital humano y la innovación se hace necesaria una clasificación interna que facilite los procesos, esto es, un modelo de gestión basado en el conocimiento para la innovación. En los tiempos actuales la Gestión del Conocimiento es el elemento central de la competitividad en las organizaciones, las mismas que orientan sus estrategias a mantener e incrementar las competencias de sus empleados, quienes desarrollan innovación. Tal es la importancia del conocimiento dentro de la organización que para lograr la productividad y competitividad se hace necesario implantar la Gerencia del Conocimiento, la que va permitir, además, la adaptabilidad de la organización a los continuos cambios del mercado. En este sentido, se evidencia que el

No hay ninguna fuente en el documento actual.Conocimiento se convierte en el activo más importante dentro de las organizaciones.

Las instituciones superiores, entre ellas las universidades, son pues las encargadas de generar, innovación y transferir conocimiento, ya que es el fin principal de éstas; por ser ellas en sí, organizaciones, también cuentan con un capital intelectual, el cual debería ser identificado, medido y evaluado. Dentro de las universidades se encuentran los centros de producción científica, entre ellos grupos de estudio, unidades de investigación, centros de investigación así como, institutos de investigación, todas ellas creadas con la finalidad de generar conocimiento é innovación y es aquí donde se encuentran los productos relacionados también con la gerencia del conocimiento.

Notándose, la necesidad de gestionar el conocimiento dentro de los centros de producción científica, es que se ha realizado el siguiente artículo, el que tiene por objetivo presentar un modelo de Gestión del Conocimiento para los centros de producción, resultado de una investigación basada en revisiones bibliográficas y valiéndonos de la experiencia de Gonzáles (2009) quien ha creado el modelo teórico para universidad públicas. Para alcanzar este objetivo, se ha iniciado este artículo con la exposición del marco teórico, para continuar con la exposición de la metodología a seguir, para identificar la situación del capital intelectual dentro de la institución, la presentación del modelo de Gestión de Conocimiento, y en el último apartado se exponen las conclusiones.

\section{MARCO TEÓRICO}

\subsection{El Conocimiento}

El conocimiento es conjunto de información almacenada producto de la experiencia, habilidades y aprendizaje, que van a permitir a las personas generar respuestas adecuadas a las situaciones que se le presenten (Bontis, 1998).

Para la teoría organizacional, el conocimiento es toda aquella información que posee valor para la organización. Esto es, información que permite generar acciones que satisfacen las demandas del mercado (Soret, 2007).

Davenport (2002), define el conocimiento como una combinación de valores, información y experiencias que permite evaluar e incorporar nuevas experiencias e información. Cabe resaltar que las empresas no crean conocimiento por sí solas, sino, son las personas que se desempeñan en ella las que crean conocimiento incorporando nuevas percepciones, experiencias que harán de la organización, una con mayor ventaja competitiva. Dentro de las organizaciones, el conocimiento se encuentra en los procesos, normas, bases de datos e incluso en documentos. 
En este orden de ideas, para las organizaciones, ya no solo empresas públicas o privadas, sino para toda organización, es importante saber con qué conocimiento cuenta la empresa, es así como se reconoce el capital intelectual. El saber cómo adquirir conocimiento, definirlo, representarlo, administrarlo y utilizarlo es un tema de gestión del conocimiento. Cabe señalar que los sistemas clásicos de contabilidad financiera no permiten una evaluación de este importante recurso para las organizaciones (Soret, 2007).

Para Soret (2007), el conocimiento tiene las siguientes características, las mismas que permiten crean ventajas competitivas en las organizaciones:

- El conocimiento es personal, es decir se origina y reside en las personas como resultado de sus experiencias personales, por ello, la imitación del conocimiento es complicada.

- Es considerado un bien que no se consume, por lo tanto, su utilización puede repetirse, a diferencia del uso de otros bienes físicos.

- El conocimiento se convierte en una guía, que permite a las personas saber los pasos a seguir en diferentes situaciones.

- Desde el punto de vista organizacional, el conocimiento permite identificar lo que el cliente necesita, valora y que esté dispuesto a pagar por ello.

\subsection{Capital Intelectual}

El Capital Intelectual es un elemento importante en la organización, y que se centra en los trabajadores. Está constituido por la experiencia, el conocimiento, la destreza y capacidades de los trabajadores, lo que les permite generar valor para la organización. Estos elementos conforman un conjunto de activos intangibles, los mismos que generalmente no se muestran en los estados contables habituales. El Capital Intelectual está compuesto por el capital humano, capital estructural y el capital relacional (Sveiby, 1997).

\section{- Capital Humano:}

Compuesto por las competencias, actitudes, valores y la agilidad intelectual de los trabajadores. Cabe resaltar que el Capital Humano, no es propiedad de la organización sino de los propios trabajadores, al irse un trabajador de la organización se lleva consigo su conocimiento; de allí el interés de las empresas del sector privado de proteger y mantener para la organización al trabajador que le genere valor (Sveiby, 1997)

\section{- Capital Estructural:}

Se denomina así, al conocimiento que la empresa ha podido internalizar y que está conformado por la cultura empresarial, los métodos de trabajo, bases de datos, software, entre otros y, que son propiedad de la empresa, las mismas que pueden protegerse legalmente (Bontis, 1998)

\section{- Capital Relacional:}

Conformada por las relaciones que aportan valor a la empresa, como la agenda de clientes, proveedores, accionistas, bancos, acuerdos, alianzas estratégicas, imagen de la empresa, entre otros (Bontis, 1998)

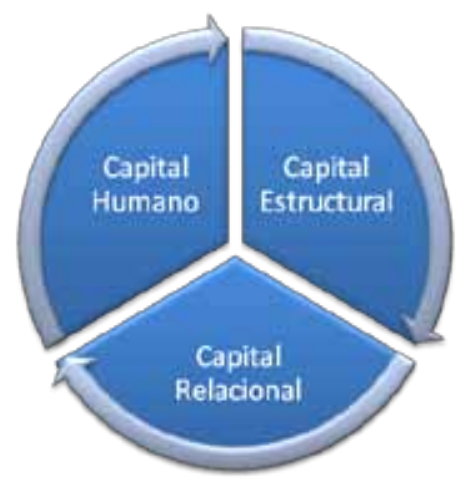

Figura No 1 Estructura del Capital Intelectual 
Estos tres tipos de capital son las dimensiones que conforman el Capital Intelectual y que permiten la innovación de productos y servicios (Soret, 2007); de modo que cualquier estrategia para gestionar el conocimiento debe tener en cuenta estas tres dimensiones de manera conjunta y no individualmente. El reto de las organizaciones es expandir la inteligencia, que es requisito para crear conocimiento; $y$, fomentar innovación.

\section{Valoración del Capital Intelectual}

El nuevo reto de las empresas para adaptarse al mercado globalizado, es gestionar y desarrollar la capacidad intelectual de sus trabajadores, a fin que éstos generen valor económico. Por lo tanto, los intangibles, constituídos por las habilidades y creatividad de los trabajadores, se convierten en el activo más valioso de las empresas, esto es, el capital intelectual se convierte en el elemento central de la organización, ya que éste contribuye a darle ventajas competitivas dentro del mercado (Román, 2005).

El capital intelectual, que posee conocimiento útil el cual aporta beneficios a la empresa, ya sea en la creación de nuevos productos o procesos, se valora sistemáticamente y de acuerdo a las expectativas de la empresa. Esta valoración se basa en el conocimiento, habilidad y creatividad del trabajador, pero, además toma en cuenta el hacer. En este sentido, para las empresas ya deja de ser importante producir en serie y masivamente, hoy la empresa moderna requiere que el conocimiento se encuentre difundido entre todos sus trabajadores para lograr una economía competitiva.

Esto quiere decir que, los recursos humanos son un factor determinante de la competitividad de la empresa. Ello acentúa la división del trabajo, separando a los trabajadores que tienen acceso al conocimiento, aportan, innovan y se adaptan a los cambios del mer- cado, de aquellos que no pueden hacerlo; generando una competencia entre los trabajadores por la aplicación de su conocimiento. A su vez, la empresa busca captar trabajadores calificados, conservarlos y protegerlos a fin que otras empresas competidoras no los sustraigan, asegurando su competitividad. Desde este escenario, el trabajador debe demostrar su capacidad de aportar soluciones e innovaciones a la empresa, para que ésta realmente valore sus conocimientos.

\subsection{Gestión del Conocimiento}

El modelo del proceso de producción estaba basado en la organización en la que el trabajador se dedicaba a producir de manera masiva y automática, bajo este sistema no era tomado en cuenta la creatividad, ni la iniciativa del trabajador constituyéndose en un objeto parte del sistema productivo, autómata y ejecutor de las decisiones del gerente (Salazar, 2004).

Con el transcurrir del tiempo y ante los cambios del mercado debido a la globalización, el empresario incorpora nuevos modelos de conducir el recurso humano, es decir, la gerencia del conocimiento, esto, basándose en que el trabajador puede agregar mayor valor a la empresa. La gerencia del conocimiento, que ordena y mide el conocimiento para alcanzar las metas y objetivos de la empresa a fin de ser organizaciones competitivas e inteligentes. Para alcanzar esta meta, se hace necesario convertir el conocimiento en activos de la organización. A continuación se presentaran algunos conceptos sobre gerencia del conocimiento.

\section{Conceptos sobre Gestión del Conoci- miento}

Para Lejter (2003), la gerencia del conocimiento es el proceso continuo de conocer, aprender, distribuir y utilizar la información 
para añadir valor y ventaja competitiva a la organización. La meta de la gerencia del conocimiento es promover la capacidad intelectual de la empresa entre quienes toman las decisiones diarias, es decir entre los trabajadores quienes finalmente determinaran el éxito o fracaso de un negocio. El conocimiento hoy por hoy, es considerado como uno de los activos más valiosos entre las grandes empresas. En este orden de ideas, la gerencia del conocimiento, permite incrementar el capital intelectual de una organización.

La gerencia del conocimiento también se comprende como el proceso que hace uso del conocimiento, para el aprovechamiento de los recursos intangibles existentes en la empresa. Bajo esta óptica, la gestión del conocimiento tiene como meta el crear una sinergia y empatía exitosa, como estrategia para promover confianza, iniciativa, autocontrol y adaptabilidad del trabajador (De la Calle y Ortiz, 2007).

Para el caso de la investigación universitaria, González (2009) define como Gestión del Conocimiento al proceso de creación, captura, distribución, compartición, asimilación, explotación uso y renovación del conocimiento como elemento generador del valor agregado en las organizaciones, a fin de hacerlas más competitivas.

Los procesos, las tecnologías de la información y los trabajadores, son componentes directamente ligados al concepto de Gerencia del Conocimiento. Esta relación nos lleva a reconocer, que existe una alianza entre el conocimiento humano y la inteligencia, basada en la tecnología, que permite que el conocimiento sea analizado e interpretado por la mente humana.

El éxito de la Gerencia del Conocimiento, por consiguiente, radica en la obtención de iniciativas que lleven a solucionar problemas, productos y servicios nuevos o mejorados que brinden a la empresa ventajas competitivas.

\section{El Conocimiento como Modelo de Gerencia}

La historia demuestra que se ha pasado de la producción en masa a la producción basada en la creatividad e innovación que va a la par con los cambios del entorno, es decir, las nuevas exigencias competitivas han desfasado al modelo de producción tradicional, la tendencia de las organizaciones hoy en día es introducir nuevos modelos de gestión, entre ellos, la Gerencia del Conocimiento. Con los modelos tradicionales, las necesidades y exigencias de los clientes eran resueltas por los dueños y gerentes de las empresas, hoy en día, este modelo ha sido desplazado por modelos enfocados hacia la obtención de mayor valor agregado, dejando en mano de los trabajadores la captación e interpretación de las necesidades de los clientes.

El modelo de gerencia del conocimiento implica la trasformación del conocimiento a través de: la socialización, combinación, externalización e internalización. Al respecto, Salazar (2004) define la socialización como el conocimiento que nace de los individuos en forma de conocimiento tácito. En cuanto a la combinación, se refiere a que el conocimiento está accesible a cualquier miembro de la empresa lo que permite generar nuevas ideas. La externalización articula los conocimientos tácitos con los implícitos; en tanto que la internalización se refiere al conocimiento explícito que se convierte en rutinario dentro de la empresa.

La transformación del conocimiento queda en manos de la organización, que tiene la misión de adiestrar y capacitar a sus trabajadores para lograr identificar y retener el conocimiento así como su aplicación en el momento que lo necesite, facilitando de esta manera, la efectiva y eficiente generación del nuevo conocimiento, lo que permitirá inno- 
var, mejorar los procesos y los productos. En este sentido, las empresas buscan trabajadores que cumplan ciertas características, entre ellas: capacidad de innovación, adaptación a los cambios, acceso a la información, conocimiento sobre adelantos tecnológicos, creativos, polivalentes y multifuncionales. Buscando captar un capital intelectual que, contribuya a garantizar a la empresa su posicionamiento en el mercado, así como, la eficiencia, productividad y competitividad. En este sentido, la empresa tiene el reto de comprometer al trabajador con las metas de la organización, haciéndolo partícipe en la toma de decisiones, concibiendo al trabajador como un sujeto estratégico, generador de respuestas oportunas, capaz de proyectar sus capacidades, basándose en su conocimiento de la realidad de la empresa y de su entorno (Hernández, 2006).

El enfoque del modelo de Gestión de Conocimiento según Maisch (2007), cubre todos los aspectos del negocio, es decir que los recursos humanos se convierten en los autores principales que van a crear e implementar los procesos de trabajo con los que se obtendrán resultados favorables. Esto quiere decir que, si una organización cuenta con personal proactivo, innovador, creativo, capacitado y además con conocimiento de las exigencias del mercado, tiene garantizada su ventaja competitiva.

La Gestión del Conocimiento, es el modelo que involucra a todos los trabajadores en la planificación, creación y aplicación de estrategias; consiguiendo que la creatividad e innovación de sus trabajadores pase a formar parte de la organización, elevando la calidad de la empresa. Esto impone al trabajador la necesidad de estar constantemente capacitándose a fin de fortalecer sus habilidades, destrezas y su creatividad para poder comprender los cambios que se generan en el mercado (Bossi, 2006).
Este modelo de gestionar el recurso humano, se ha convertido en la nueva técnica de rentabilidad y crecimiento económico de las organizaciones; de tal modo que, el empresario influye en la conciencia de los trabajadores comprometiéndolos en la necesidad de alinearse con los objetivos é intereses de la empresa.

\section{Capitalista del Conocimiento}

La Gerencia del Conocimiento acumula la producción de innovaciones que se constituyen en fuente de valor para la empresa, la que monopoliza el trabajo mental del trabajador, acumulando y apropiándose del conocimiento como capital de la empresa. Esto se resume en que el nuevo sistema de creación de riqueza se basa en el conocimiento ajeno. El empresario al contratar al trabajador, y como se ha mencionado anteriormente, demanda trabajadores capacitados y profesionales; ocasiona que, para que el trabajador sea considerado productivo, debe tener una formación y capacitación continua durante toda su vida laboral, asumiendo personalmente los gastos de la misma, y a la vez, la responsabilidad de su situación laboral, ya que si no consigue emplearse será debido a que no se encuentra suficientemente formado para el nuevo mercado laboral (Diez, 2010).

Las empresas están interesadas en el conocimiento que los trabajadores puedan aportarles, no solamente para producir con mayor calidad, sino además, para apropiarse de dicho conocimiento, ya que capitaliza los nuevos aportes brindados por los trabajadores, apoyándose en diferentes estrategias para defender los conocimientos que se producen dentro de la organización. La estrategia más utilizada para este fin son las patentes; de esta manera los nuevos productos, innovaciones, adelantos tecnológicos é 
ideas producidas por los trabajadores pasan a ser consideradas propiedad de la empresa y son patentadas a nombre de éstas. Con esta estrategia, la empresa consigue impedir reclamos por parte de los trabajadores, o que el nuevo conocimiento sea copiado por otras empresas, logrando obtener el control total del conocimiento (Iglesias, 2000).

Zorrilla (1997), considera que la Gerencia del Conocimiento debe encargarse de hacer las negociaciones entre quienes tienen conocimiento y quienes hacen uso de ésta, en esta negociación el gerente debe tener la capacidad de gestionar el tiempo y el esfuerzo de los trabajadores asumiendo modelos colaborativos, a fin de lograr que todos los trabajadores se involucren y comprometan con la organización.

\section{MODELO PARA EL DESARROLLO DE GESTIÓN DEL CONOCIMIENTO EN EL ÁREA DE INVESTIGACIÓN}

Se puede deducir que la Gestión del Conocimiento es de vital importancia para el desarrollo y subsistencia de las organizaciones, y debe ser valorado también en el ámbito académico como un elemento del quehacer universitario, en especial a lo referente a los Grupos de estudio, Unidades, Centros e Institutos de Investigación (en adelante simplemente GUCII), siento éstos, uno de los principales pilares de la misión de una universidad. Esta sección se centra en los GUCII de la Universidad Nacional Mayor de San Marcos (UNMSM), ya que es allí donde se encuentran los productos relacionados directamente con la Gestión del Conocimiento.

En esta sección se presenta un modelo teórico basado en la Gerencia del Conocimiento para los GUCII de la UNMSM, como resultado de una investigación basada en re- visiones bibliográficas, en la que se presentan los pasos necesarios para la identificación de la situación actual de los GUCII; el modelo que se presenta fue desarrollado por González (2009) para los Centros de Producción Científica de Universidades Públicas.

\subsection{Marco Institucional}

Si bien es cierto, la teoría presentada por González (2009), va dirigida a las universidades públicas, buscamos adaptarla a los GUCII de la UNMSM. La UNMSM, es una universidad pública, localizada en el departamento de Lima (Perú), conformada por 23 Facultades, con un total de 30230 alumnos; cuenta con 26 Institutos de Investigación, 4 Centros de Investigación y 3 Unidades de Investigación distribuidos en las diferentes Facultades, según lo refiere la página web oficial de la UNMSM .Cabe resaltar que la página web de la UNMSM no informa el número de grupos de estudios conformado por estudiantes $y$, que forman parte también del quehacer investigativo de la universidad.

Generalmente, estas diferentes oficinas dependen directamente de cada Decanato, como el caso de la Facultad de Ciencias Contables. Sin embargo, todas ellas rinden informes al Consejo Superior de Investigación que depende directamente del Vicerrectorado de Investigación como se muestra en el siguiente esquema (figura No 2)

\section{Tomado de la página web del Vicerrectorado de Investigación UNMSM}

En general en la UNMSM, se evidencia la falta de política institucional para impulsar la investigación basándose en la Gerencia del Conocimiento, esto pude explicarse en que, en la mayoría de los casos, la investigación en la universidad es un proceso impuesto y no una actividad que nazca propiamente 


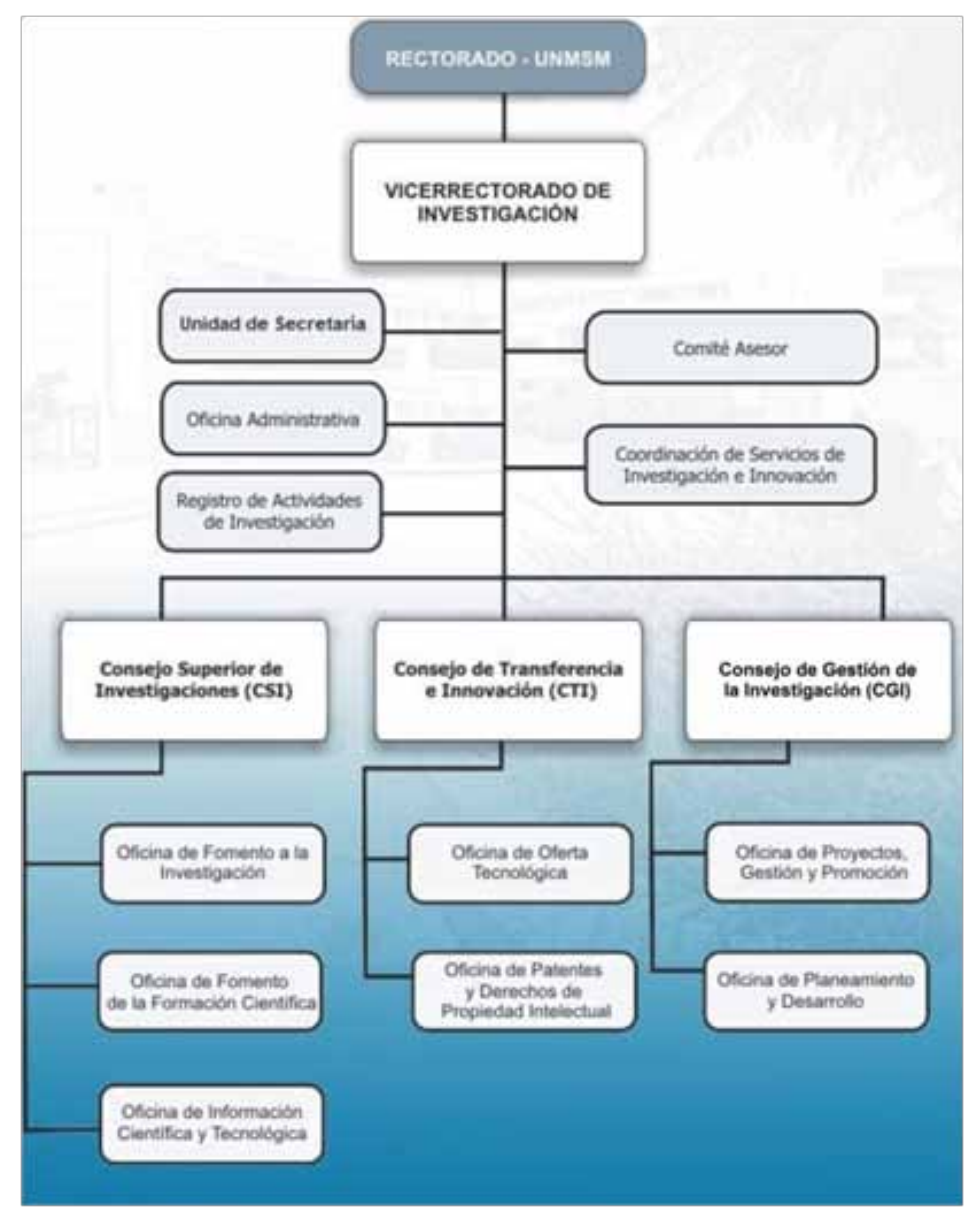

Figura No 2 Organigrama del Vicerrectorado de Investigación de la UNMSM - 2012

de los docentes, esto, sumado al bajo apoyo económico a los docentes que lo realizan, de modo que les permita desplazarse y asistir a eventos académicos, así como, pocas Facultades cuentan con lugares apropiados para reunirse, a fin de realizar trabajos propios de la investigación, es decir, carecen de espacios para la creación y socialización propiamente de conocimiento.

La motivación es un elemento importante en el aprendizaje organizacional, la falta de estímulo se ve reflejada en la baja productividad de investigación, toda vez que la investigación es considerada como un indicador para que el docente sea nuevamente contratado, o porque requiera incrementar su puntaje para mantener su categoría o contrato dentro de la universidad. De otro lado, la falta de docentes con la formación requerida para la investigación en sí y el desconocimiento por parte de la universidad respecto a su capital intelectual, son elementos que contribuyen a una deficiente producción investigativa.

\subsection{Metodología para la Gestión del Co- nocimiento}

Los pasos a seguir para identificar el estado actual de la situación del capital intelectual en los GUCII de la UNMSM, planteados por Gonzáles (2009)son los siguientes:

- Identificación de las fuentes primarias: identificar a los Directivos de los Grupos de Estudios, Unidades de Investigación, Centros de Investigación e Institutos de Investigación, con la finalidad de obtener información sobre el número de investiga- 
ciones que se desarrollan bajo su mando, así como, los nombres de los responsables de las mismas para aplicarles una futura encuesta.

- Aplicación de una encuesta: la encuesta a aplicar en los directivos y responsables de estudios de investigación estará enfocada al autodiagnóstico de estos GUCII de la UNMSM.

- Recolección de información de fuentes secundarias: referido a la consulta en base de datos, documentos e informes que nos puedan ayudar a complementar información sobre estos GUCII, entre los que deben estar los compendios estadísticos proporcionados por la Unidad de Estadística de la misma UNMSM, así como, la publicación; "Indicadores de Gestión" elaborada por la Oficina General de Planificación y la Oficina de Estadística e Informática de la UNMSM.

- Identificación de la población, en este caso ya se las tiene identificadas, y son 33 Centros de Producción Científica; sin embargo, para aplicar esta metodología será necesario además, averiguar el total de Grupos de Estudios o investigaciones que se están realizando dentro de cada Facultad.

\subsection{Variables de la Gestión del Conocimiento}

A continuación se enlista las variables de Gestión del Conocimiento enfocada a los GUCII.

\section{Variables Cualitativas:}

- Director de Centros de Producción Científica y Responsables de las Investigaciones: quienes manejan información sobre el conocimiento y capacidades de los grupos que se encuentran desarrollando investi- gación dentro de la Facultad en la que se desempeñan.

- Participantes del Grupo de Investigación: los miembros y colaboradores que proporcionarán información sobre el capital humano con el que cuentan los GUCII de la UNMSM.

- Vinculación con redes de investigación, comunidades científicas y participación en reuniones, conferencias, seminarios, etc. Esta información permitirá evaluar el Capital Relacional con el que cuentan los GUCII de la UNMSM.

- Clasificación y categoría de los estudios de investigación.

- Identificación de las bases de datos, sistemas de gestión, desarrollo de nuevas tecnologías, patentes. Permitirá identificar y evaluar el Capital Estructural con el que cuenta los GUCII de la UNMSM.

- Línea de investigación a la que pertenece el estudio.

- Lugar en el que se lleva a cabo los estudios de investigación.

- Mecanismos de Comunicación

- Frecuencia de uso de las TICs

- Nivel académico alcanzado por cada miembro participante del estudio de investigación.

\section{Variables Cuantitativas}

- Productividad Académica: referida a las cantidades verificables de nuevos conocimientos.

- Recursos financieros, utilizados en la investigación.

- Herramientas de búsqueda de información utilizada.

- Medios de difusión del producto de investigación. 


\subsection{Modelo Propuesto para la Gestión del Conocimiento}

El siguiente es un modelo teórico propuesto por González (2009), fruto del estudio de la dinámica del conocimiento dentro de la universidad. Este modelo se utiliza como soporte para el intercambio de los recursos de información y que busca mejorar la dinámica de los procesos de creación de conocimiento mediante actividades de investigación, que permitan la conversión del conocimiento de los investigadores al investigador, y lograr así, el aprovechamiento de los resultados en el proceso de transferencia de información. Para la aplicación de este modelo, es necesario tener en cuenta lo siguiente:

- Políticas investigativas institucionales, a fin de dar pertinencia a la investigación; este punto concierne al Consejo Superior de Investigación.

- Generación de cultura investigativa. Es decir, que los docentes sean investigadores.

- Generación de una red interna que permita la conexión entre los grupos de investigación.
- Incentivar y facilitar la publicación de artículos en revistas indexadas

El modelo propuesto por Gonzáles (2009), es cíclico, se inicia con la entrada de una serie de insumos, que ha demostrado que son elementos importantes en la Gestión del Conocimiento para los Centros de Producción de Investigación: Red interna, internet de banda ancha, recursos que permitan la conectividad con otras universidades nacionales y extranjeras, presupuesto de inversión para la investigación que debe incluir presupuesto de inversión para la capacitación del investigador. Estos elementos de entrada, sufren una transformación a través de los recursos humanos y físicos entre los que destacan la participación en convocatorias, ponencias, artículos en revistas indexadas, ferias científicas y tecnológicas por programa académico; para obtener como resultado la recategorización de la investigación, valor del capital intelectual, interacción con el sector productivo, nuevos grupos de investigación, productividad científica, reconocimiento de comunidades y redes, é incluso acreditación institucional y de programas.

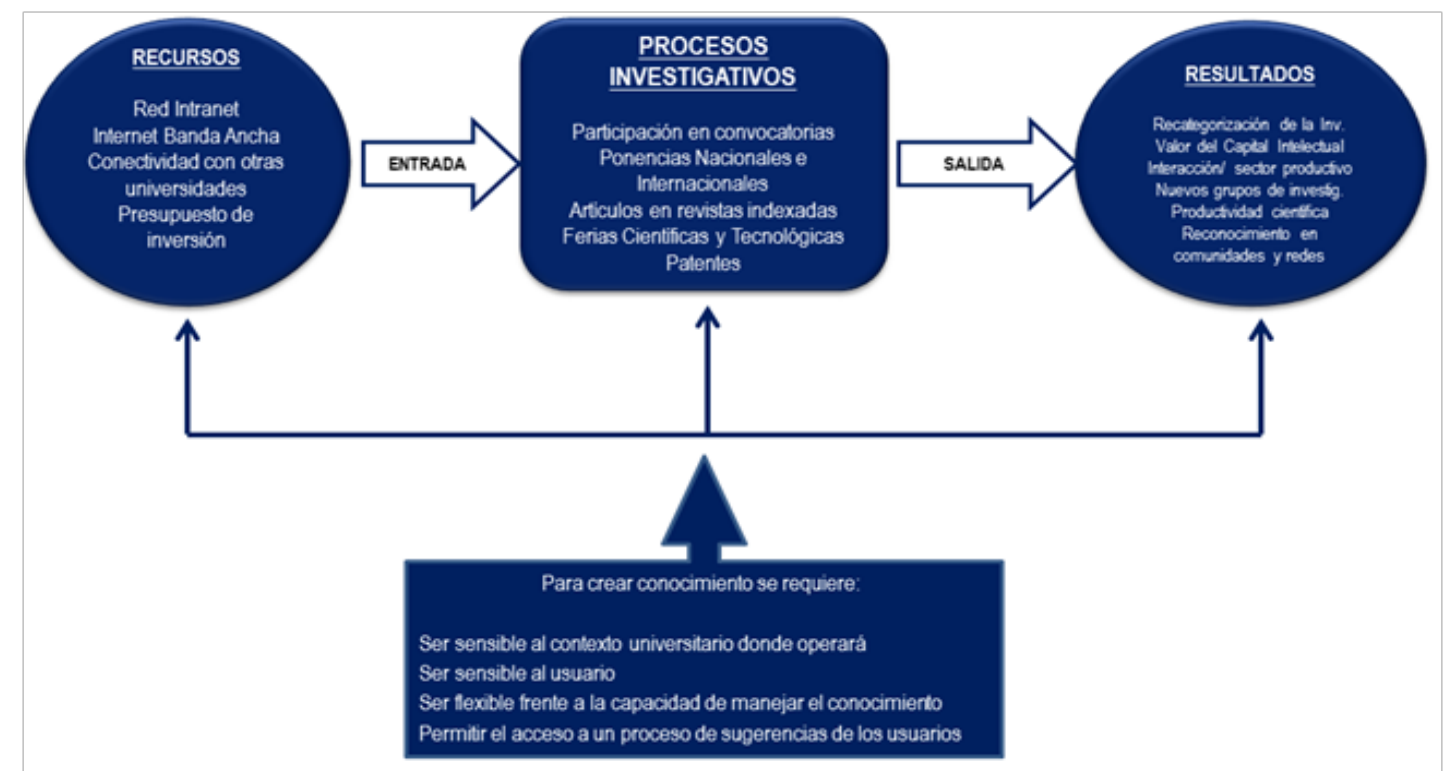

Figura No 3 Modelo de Gestión de Conocimiento propuesto por Gonzáles (2009) para Centros de Investigación. 
Este modelo de gestión de conocimiento, aunque teórico, sirve para la creación, utilización, difusión y transferencia de conocimientos entre los Centros de Producción Científica y evidencian los beneficios de la tecnología actual ya que permiten alcanzar mayor grado de eficiencia en la investigación, generando ventajas competitivas frente a otras organizaciones, en este caso, frente a otras universidades públicas y privadas.

\section{CONCLUSIONES}

La economía globalizada ha generado los principales cambios en los procesos productivos y en la concepción del trabajador, desarrollándose la gerencia del conocimiento como el modelo más idóneo para disponer del capital intelectual que se constituye hoy en día, como el activo más importante de las empresas.

El recurso humano está considerado por la nueva estructura organizacional, como factor fundamental en el desarrollo de empresas competitivas, convirtiéndose el conocimiento en la nueva fuente de riqueza, considerada el activo más valioso de la organización, ya que genera ventajas competitivas.

La Gerencia del Conocimiento, como modelo de gestión, es un proceso que permite que los empresarios tengan el control total del capital intelectual, por ello los empresarios buscan fuerza laboral que le representen ventajas competitivas y beneficios a corto plazo.

La Gestión del Conocimiento debe ser valorado en el ámbito académico, ya que es una organización que por sus funciones $y$ misión, se encarga de generar conocimiento e innovación, por ello se hace uso del marco teórico para presentar y explicar un modelo para la consolidación de la Gestión del Co- nocimiento en los GUCII de la UNMSM, orientada al mejoramiento continuo de estos centros de producción científica. Es importante destacar que, es necesario ser flexible en las universidades para poder adaptarse rápidamente a los cambios tecnológicos y demás transformaciones que trae consigo la globalización.

\section{REFERENCIAS BIBLIOGRÁFICAS}

1. Bossi Queiroz, A. (2006). El capital intelectual en el sector público. Sociedade, Contabilidade e Gestao, 1(1), 5-18.

2. Bontis, N. (1998). Intellectual capital: An exploratory study that develops measures and models. Management Decision, 32(2), 63-76.

3. Davenport, T., \& Glaser, J. (2002). Just in time delivery comes to knowledge management. Harvard Business Review, Julio, 5-9.

4. De la Calle Durán, C., \& Ortiz de Urbina Criado, M. (2007). Los modelos de capital intelectual en las empresas españolas: Una aplicación práctica. Entelequia. Revista Interdisciplinar, (3), 185-201.

5. Diéz Gutierrez, E. (2010). Globalización neoliberal y sus repercusiones en la educación. Revista Electrónica Interuniversitaria de Formación del Profesorado, 13, 23-38.

6. González Millán, J. J. (2009). Modelo para el desarrollo de la gestión del conocimiento en los centros de investigacion de las universidades públicas colombianas. Gestión y Estrategia, 35(Enero-Junio), 47-62.

7. Hernández López, M. (2006). La gestión del conocimiento un nuevo enfoque parar liderar los cambios en la nueva econo- 
mía | Pág. Web: Gestiopolis. Consultado junio 13, 2012. http://www.gestiopolis. com/canales7/ger/gestion-del-conocimiento-para-liderar-el-cambio.htm.

8. Iglesias Fernández, J. (2000). El trabajo general como justificacion de la renta básica. Pág. Web: Attac Madrid. Consultado mayo 12, 2012.www.attacmadrid. org/d/1/el_trabajo_general.htm.

9. Lejter, N. (2003). Gerencia del conocimiento: Moda o revolución? Pág. Web: De Gerencia. Consultado mayo 10, 2012.

10. http://www.degerencia.com/articulo/ gerencia_del_conocimiento

11. Maisch Molina, E. (2007). La alineación estratégica de los recursos humanos a la gestión organizacional. Pág. Web: Mujeres de Empresa. Consultado junio 21, 2012. http://www.mujeresdeempresa. com/relaciones_humanas/070901alineacion-estrategica-de-los-recursoshumanos.asp.

12. Román, N. (2005). Capital intelectual: Generador de éxito en las empresas. Revista Visión Gerencial, 3(3), 67-79.

13. Salazar, J. M. (2004). Algunas reflexiones sobre la gestión del conocimiento en las empresas. Intangible Capital, 2, 129-134.
14. Soret Los Santos, I. (2007). Modelo de Medición de Conocimiento y Generación de Ventajas Competitivas sostenibles en el ámbito de la iniciativa «Respuesta Eficiente al Consumidor». Tesis Doctoral. Universidad Rey Juan Carlos. Facultad de Ciencias Jurídicas y Sociales, España.

15. Sveiby, K. (1997). The invisible balance sheet: Key indicators for accounting, control and evaluation of Know-How companies. The Konrad Group. Suecia

16. UNMSM. (2012). Vicerrectorado de Investigación. Pág. Web: Universidad Nacional Mayor de San Marcos. Consultado junio 21, 2012. http://vrinvestigacion.unmsm.edu.pe/

17. UNMSM. (2012). Universidad Nacional Mayor de San Marcos. Pág. Web: Universidad Nacional Mayor de San Marcos. Consultado junio 21, 2012. http://www. unmsm.edu.pe

18. Zorrilla, H. (1997). La gerencia del conocimiento y la gestión tecnológica. SHT. Promoviendo el desarrollo del talento humano. Pág. Web: SHT. Consultado junio 21, 2012. http://www.sht.com. ar/archivo/Management/conocimiento.htm 